\title{
Development of tricuspid regurgitation late after left-sided valve surgery: A single-center experience with long-term echocardiographic examinations
}

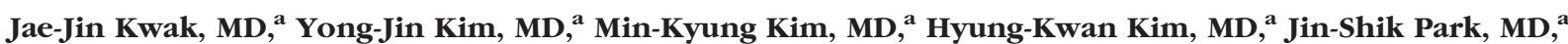

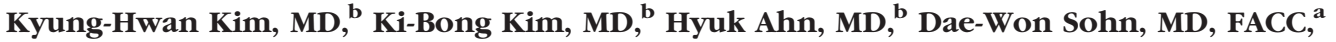
Byung-Hee Oh, MD, FACC, ${ }^{\mathrm{a}}$ and Young-Bae Park, MD ${ }^{\mathrm{a}}$ Seoul, Soutb Korea

Objectives This study sought to investigate the incidence and identify the predictors of significant tricuspid regurgitation (TR) development long after left-sided valve surgery.

Methods Of 615 patients who underwent surgery for left-sided valve disease between 1992 and 1995, 335 patients without significant TR who completed at least 5 years of clinical and echocardiographic follow-up were enrolled. Late significant TR development was assessed by echocardiography with a mean follow-up duration of $11.6 \pm 2.1$ years.

Results Significant late TR was found in 90 patients (26.9\%). Patients with late TR showed an advanced age $(47.6 \pm$ 13.4 vs $44.3 \pm 13.2$ years, $P=.04)$, a higher prevalence of preoperative atrial fibrillation $(83.3$ vs $46.5 \%, P<.001)$, a greater left atrial dimension $(56.9 \pm 13.2 \mathrm{vs} 52.4 \pm 11.5 \mathrm{~mm}, P=.006)$, and a higher prevalence of prior valve surgery (40.0 vs $25.3 \%, P=.01)$. In addition, late TR occurred more frequently in patients who had undergone mitral valve surgery than in those who did not $(93.3$ vs $72.2 \%, P<.001)$. However, multivariate analysis showed that the presence of preoperative atrial fibrillation (odds ratio $5.37 ; 95 \% \mathrm{Cl} 2.71-10.65 ; P<.001$ ) was the only independent factor of late TR development. Patients who developed late TR had a lower event-free survival rate than those who did not $(P=.03)$.

Conclusions The development of significant TR long after left-sided valve surgery is not uncommon with an estimated incidence of $27 \%$ and is closely associated with a poor prognosis. The presence of preoperative atrial fibrillation was identified as the only independent predictor of the development of late TR. (Am Heart J 2008;155:732-7.)

Tricuspid regurgitation (TR) is a common finding in patients undergoing mitral or combined mitral/aortic valve surgery. ${ }^{1,2}$ Although TR may decrease gradually after left-sided valve surgery owing to reduced right ventricular pressure or volume overload, ${ }^{3}$ TR does not always regress after adequate correction of the underlying lesions ${ }^{4,5}$ and often progresses some time after surgery without leftsided valvular dysfunction, even after tricuspid annuloplasty. Furthermore, TR can appear de novo during postoperative follow-up. Because it is well known that TR progression badly affects long-term mortality and morbidity, and that surgical correction of TR after left-sided valve surgery is associated with disappointingly high

From the ${ }^{a}$ Department of Internal Medicine, Seoul National University Hospital, Seoul, South Korea, and ${ }^{b}$ Department of Thoracic Surgery, Seoul National University College of Medicine, Seoul National University Hospital, Seoul, South Korea.

Submitted March 15, 2007; accepted November 9, 2007.

Reprint requests: Yong-Jin Kim, MD, Division of Cardiology, Department of Internal Medicine, Seoul National University College of Medicine, 28 Yongon-dong, Chongno-gu, Seoul, 110-744, South Korea.

E-mail: kimdamas@snu.ac.kr

0002-8703/\$ - see front matter

(c) 2008, Mosby, Inc. All rights reserved.

doi:10.1016/i.ahj.2007.11.010 mortality and morbidity, ${ }^{6-9}$ the prevention of late TR is clinically important. In this regard, information regarding the incidence and predictors of progression or development of late TR is of substantial value for determining optimal operative timing and the surgical strategy to prevent late TR development, at the time of surgery for underlying left-sided valve disease. Several previous reports on this topic have been limited by a relatively small study population, ${ }^{5,10}$ a short-term follow-up, ${ }^{11}$ or the lack of reliable echocardiographic data. ${ }^{12}$ Therefore, the present study was performed to investigate the incidence of late de novo TR development after left-sided valve surgery and to identify preoperative predictors in patients without significant preoperative TR by long-term clinical and echocardiographic follow-up.

\footnotetext{
Methods

Study population

This study was based on our consecutive experiences of patients who underwent surgical correction for left-sided valve disease from January 1992 to December 1995. During this period, a total of 615 patients underwent surgery for mitral, aortic, or combined mitral/aortic valve disease at our institution,
} 
Table I. Baseline characteristics of the study subjects

\begin{tabular}{|c|c|}
\hline Characteristics & n (\%) \\
\hline Sex (male) & $159(47.5)$ \\
\hline Age (y) & $45.2 \pm 13.3$ (range $16-81)$ \\
\hline BMI $\left(\mathrm{kg} / \mathrm{m}^{2}\right)$ & $21.4 \pm 2.8$ (range 14.5-29.4) \\
\hline Duration of follow-up (y) & $11.6 \pm 2.1$ (range 61-175) \\
\hline \multicolumn{2}{|l|}{ Preoperative electrocardiography } \\
\hline Sinus rhythm & $146(43.6)$ \\
\hline Atrial fibrillation & $189(56.4)$ \\
\hline \multicolumn{2}{|l|}{ Preoperative echocardiography } \\
\hline LV Ejection fraction (\%) & $60.1 \pm 12.6$ (range 20-89) \\
\hline LV end-diastolic diameter (mm) & $57.3 \pm 11.9$ (range $35-100)$ \\
\hline Left atrial dimension $(\mathrm{mm})$ & $53.7 \pm 12.1$ (range 20-104) \\
\hline \multicolumn{2}{|l|}{ Preoperative cardiac catheterization } \\
\hline $\begin{array}{l}\text { Pulmonary artery systolic } \\
\text { pressure }(\mathrm{mm} \mathrm{Hg})\end{array}$ & $42.9 \pm 18.6$ (range $8-140$ ) \\
\hline $\begin{array}{l}\text { Pulmonary capillary wedge } \\
\text { pressure }(\mathrm{mm} \mathrm{Hg})\end{array}$ & $20.0 \pm 8.8$ (range $3-50$ ) \\
\hline Mean right atrial pressure $(\mathrm{mm} \mathrm{Hg})$ & $5.8 \pm 4.0$ (range 1-18) \\
\hline
\end{tabular}

$B M I$, Body mass index; $L V$, left ventricle.

and these were initially considered eligible for this study. However, we excluded patients who underwent combined tricuspid valve replacement $(n=19)$, aortic surgery $(n=20)$, correction of combined congenital anomaly $(\mathrm{n}=31)$, and coronary artery bypass graft surgery $(n=22)$. Seventy-four patients who were lost to follow-up, 40 patients who died within 5 years of index surgery, and 74 patients with significant preoperative TR, moderate or severe in degree, were also excluded. Accordingly, 335 patients (159 male, 47.5\%) who completed at least a 5-year clinical and echocardiographic follow-up were enrolled.

\section{Baseline data procurement}

Patient hospital records and our cardiac surgery database were reviewed for the following: age at index surgery, sex, body weight, height, heart rhythm, type of surgery, and history of prior cardiac surgery. Preoperative cardiac catheterization was performed in all patients, and parameters including pulmonary capillary wedge pressure, systolic pulmonary artery pressure, and mean right atrial pressure were measured. A preoperative echocardiographic examination was performed in all patients by a skilled echocardiographer and interpreted by an experienced cardiologist. Left ventricular end-systolic and end-diastolic diameters, left ventricular ejection fraction, left atrial dimension, and valvular functions were comprehensively evaluated.

Etiologies of valvular disease were confirmed based on surgical observations and/or pathologic findings. The study protocol was approved by the Institutional Review Board of Seoul National University Hospital.

\section{Echocardiographic examinations}

Comprehensive 2-dimensional and Doppler echocardiographic examinations were performed preoperatively and repeated after discharge from hospital by skilled echocardiographers in a standard manner using commercially available echocardiographic devices. The presence of TR and its severity were assessed using multiple transthoracic windows. The maximal TR jet area in any echocardiographic view was used to semi-quantitatively estimate TR grade using a standard color
Table II. Underlying valve diseases at time of surgery

\begin{tabular}{ccccccc} 
& & \multicolumn{4}{c}{ AV diseases } & \\
\cline { 3 - 6 } & & Normal & AS & AR & ASR & Total \\
\hline \multirow{2}{*}{ MV diseases } & Normal & 0 & 18 & 30 & 16 & 64 \\
& MS & 50 & 2 & 16 & 12 & 80 \\
& MR & 62 & 3 & 36 & 8 & 109 \\
& MSR & 46 & 2 & 16 & 18 & 82 \\
& Total & 158 & 25 & 98 & 54 & 335 \\
\hline
\end{tabular}

$M V$, Mitral valve; $M R$, mitral regurgitation; $M S$, mitral stenosis; $M S R$, combined mitral stenosis and regurgitation; $A V$, aortic valve; $A S$, aortic stenosis; $A R$, aortic regurgitation; $A S R$, combined aortic stenosis and regurgitation.

Doppler technique. Tricuspid regurgitation grade was classified as none, trace, mild, moderate, or severe in each patient. ${ }^{13,14}$ To simplify statistical analysis, significant TR was defined as moderate or severe in degree. Maximal TR jet velocity was obtained by continuous-wave Doppler and was used to calculate systolic pulmonary artery pressure. Latest echocardiographic examinations were regarded as the end of the follow-up period.

\section{Statistical analysis}

Continuous variables are expressed as means $\pm \mathrm{SD}$, and categorical variables as percentages. Multiple logistic regression analysis using the forward stepwise selection process, including all significant parameters identified by univariate analysis and previously known confounding variables, was undertaken to identify parameters independently associated with the presence of significant TR after surgery. Event-free cumulative survival rates were plotted using the Kaplan-Meier method and comparisons were made between patient groups with and without late significant TR using the log-rank test. SPSS 13.0 (SPSS Inc, Chicago, IL) was used for the statistical analyses and $P$ values of $<.05$ were considered statistically significant.

\section{Results}

\section{Baseline characteristics}

The baseline characteristics of the 335 study subjects are summarized in Table I. Mean follow-up duration was $11.6 \pm 2.1$ years (range 5.1-14.6 years). Preoperative electrocardiograms showed atrial fibrillation in 189 (56.4\%) of the patients.

Mitral valve surgery (mitral valve replacement or mitral valve repair) without accompanying aortic valve surgery was performed in 174 patients (51.9\%), aortic valve surgery alone (aortic valve replacement or aortic valve repair) in $74(22.1 \%)$, and combined mitral and aortic valve surgery in the remaining 87 (26.0\%). At the time of left-sided valve surgery, tricuspid annuloplasty was performed in 15 patients (4.5\%).

Preoperative diagnoses according to valve disease and the procedures performed to correct underlying valve abnormalities are detailed in Tables II and III, respectively. Of 261 patients who underwent mitral valve surgery with or without an aortic valve operation, mitral valve diseases were caused by rheumatic disease in 138 (52.9\%), 
Table III. Types of left-sided valve surgery

\begin{tabular}{|c|c|c|c|c|c|}
\hline & \multicolumn{3}{|c|}{ AV surgery } & \multirow[b]{2}{*}{ Total } \\
\hline & & AVR & $\begin{array}{l}\text { AV } \\
\text { repair }\end{array}$ & $\begin{array}{l}\text { Without } \\
\text { AV surgery }\end{array}$ & \\
\hline \multirow[t]{4}{*}{ MV surgery } & $M V R$ & 77 & 1 & 133 & 211 \\
\hline & MV repair & 7 & 2 & 41 & 50 \\
\hline & $\begin{array}{l}\text { Without MV } \\
\text { surgery }\end{array}$ & 69 & 5 & 0 & 74 \\
\hline & Total & 153 & 8 & 174 & 335 \\
\hline
\end{tabular}

MVR, Mitral valve replacement; $A V R$, aortic valve replacement.

\section{Figure 1}

\section{All patients ( $n=335$}

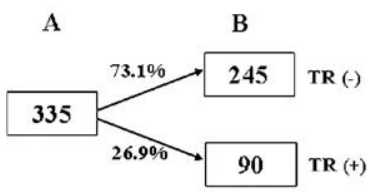

Preoperative Af ( $n-189)$

A

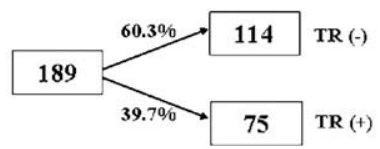

Preoperative sinus rhythm $(n=146)$

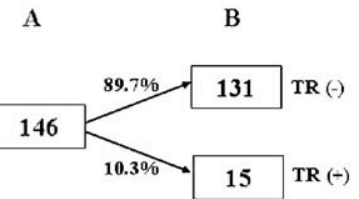

Development of significant TR during follow-up after left-sided valve surgery in all study subjects, and in patients according to cardiac rhythm. Af, Atrial fibrillation.

prosthetic valve failure in 77 (29.5\%), mitral valve prolapse in $26(10.0 \%)$, and others (including endocarditis and congenital disease) in 19 (7.3\%). On the other hand, of the 161 patients who underwent aortic valve surgery with or without a mitral valve operation, degenerative disease was the most common etiology for aortic valve surgery ( 49 patients, $30.4 \%$ ), followed by rheumatic disease in 46 (28.6\%), prosthetic valve failure in $33(20.5 \%)$, and others (including congenital disease and endocarditis) in $33(20.5 \%)$.

\section{Development of late significant TR}

The development of late significant TR in all study subjects and patients according to cardiac rhythm is shown in Figure 1. Significant TR had developed in 90 patients (26.9\%) at last follow-ups, and this was severe in 25 (7.5\%).

Clinical and echocardiographic characteristics according to the presence or absence of late TR are summarized in Table IV. Patients who developed late TR were older $(47.6 \pm 13.4$ vs $44.3 \pm 13.2$ years, $P=.04)$, had a higher prevalence of preoperative atrial fibrillation $(83.3 \% \mathrm{vs}$ $46.5 \%, \chi^{2}=36.3, P<.001$ ), a greater left atrial dimension (56.9 \pm 12.3 vs $52.4 \pm 11.5 \mathrm{~mm}, P=.006)$, a lower left

Table IV. Main clinical and echocardiographic characteristics according to the presence of significant late TR

\begin{tabular}{lclc} 
Characteristics & $\begin{array}{c}\text { Late TR (+) } \\
(\mathbf{n}=\mathbf{9 0})\end{array}$ & $\begin{array}{c}\text { Late TR (-) } \\
(\mathbf{n}=\mathbf{2 4 5})\end{array}$ & P Value \\
\hline Age (y) & $47.6 \pm 13.4$ & $44.3 \pm 13.2$ & .04 \\
Male/female & $38: 52$ & $121: 124$ & .24 \\
BMl (kg/m) & $21.3 \pm 2.6$ & $21.5 \pm 2.9$ & .63 \\
MV/non-MV surgery & $84: 6$ & $177: 68$ & $<.001$ \\
Previous valve surgery & $36(40.0 \%)$ & $62(25.3 \%)$ & .009 \\
Preoperative AF & $75(83.3 \%)$ & $114(46.5 \%)$ & $<.001$ \\
Preoperative & & & \\
$\quad$ echocardiographic data & & & \\
$\quad$ LVEF (\%) & $60.1 \pm 13.0$ & $60.0 \pm 12.5$ & .98 \\
$\quad$ LVEDD (mm) & $54.6 \pm 11.1$ & $58.4 \pm 12.0$ & .01 \\
$\quad$ LA size (mm) & $56.9 \pm 13.2$ & $52.4 \pm 11.5$ & .006 \\
Preoperative cardiac & & & \\
$\quad$ catheterization data & & & \\
PASP (mm Hg) & $44.6 \pm 16.9$ & $42.2 \pm 19.2$ & .34 \\
PCWP (mm Hg) & $20.8 \pm 8.9$ & $19.7 \pm 8.7$ & .36 \\
mRAP (mm Hg) & $5.8 \pm 3.8$ & $5.9 \pm 4.0$ & .94 \\
\hline
\end{tabular}

*Significant late TR was defined as moderate or severe in degree. AF, Atrial fibrillation; $L V E F$, left ventricular ejection fraction; LVEDD, left ventricular end-diastolic dimension; $L A$, left atrium; $P A S P$, pulmonary artery systolic pressure; $P C W P$, pulmonary capillary wedge pressure; $m R A P$, mean right atrial pressure.

ventricular end-diastolic dimension ( $54.6 \pm 11.1$ vs $58.4 \pm$ $12.0 \mathrm{~mm}, P=.01$ ), and were more likely to have undergone prior valve surgery ( $44.0 \%$ vs $25.3 \%, \chi^{2}=6.9$, $P=.009)$. Of note, late TR was observed more frequently in patients with mitral valve surgery $\left(93.3 \%\right.$ vs $72.2 \%, \chi^{2}=$ $17.0, P<.001)$. However, other factors, such as etiologies, left ventricular ejection fraction, systolic pulmonary artery pressure, pulmonary capillary wedge pressure, and mean right atrial pressure, were comparable in the 2 study groups.

\section{Independent determinants of late TR}

To identify independent clinical and echocardiographic factors for the development of late TR, we performed multivariate forward conditional logistic regression analysis using clinical, echocardiographic, and cardiac catheterization parameters, that is, age, sex, history of previous valve surgery, performance of mitral valve surgery, presence of preoperative atrial fibrillation, left atrial size, left ventricular end-diastolic dimension, preoperative systolic pulmonary artery pressure, and presence of preoperative mild TR. This analysis identified the presence of preoperative atrial fibrillation (odds ratio $5.37,95 \%$ CI $2.71-10.65, P<.001)$ as the only independent determinant of the development of late TR.

\section{Clinical events during follow-up}

Of the 335 study subjects, 62 patients succumbed away during follow-up. Causes of death were heart failure in 9, cerebrovascular events in 13 , myocardial infarction in 5 , endocarditis in 12 , malignancy in 5 , unexpected 


\section{Figure 2}

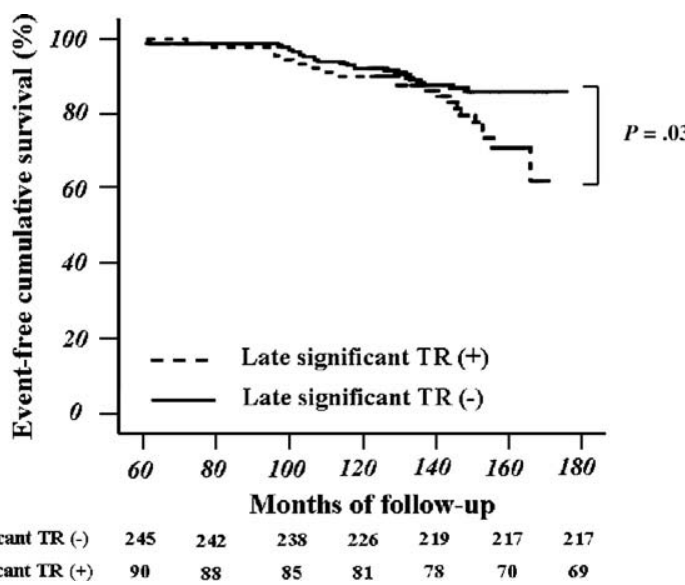

Comparison of event-free cumulative survival rates according to the presence or absence of late significant tricuspid regurgitation.

accidents in 2 , liver disease in 5 , sepsis in 2 , other causes including sudden death in 3, and unknown in 6 . During the same period, reoperation for isolated severe TR was performed in 12 patients, among whom 2 died from right ventricular failure. When cardiovascular death and reoperation were defined as clinical events, the probability of event-free survival rate in patients without late TR was $97.0 \% \pm 1.1 \%$ at 100 months, $87.7 \% \pm 2.8 \%$ at 140 months, $85.9 \% \pm 2.6 \%$ at 160 months, and $85.9 \% \pm$ $2.6 \%$ at 175 months. By comparison, the probability of event-free survival rate in patients with late TR was $94.4 \% \pm 2.4 \%$ at 100 months, $86.2 \% \pm 3.7 \%$ at 140 months, $70.9 \% \pm 5.9 \%$ at 160 months, and $62.0 \% \pm 9.8 \%$ at 175 months. Thus, patients with late TR showed significantly lower event-free survival rates during followup $(P=.03)$ (Figure 2).

\section{Discussion}

In the present study, it was found that the incidence of late TR was $26.9 \%$ after left-sided valve surgery in patients without preoperative significant TR, and that the incidence of severe TR was $7.5 \%$. Multivariate logistic regression analysis revealed the presence of preoperative atrial fibrillation as the only independent predictor of late TR development, with an approximately 5-fold elevated risk. Furthermore, late TR was found to be accompanied by a lower long-term event-free survival rate. To the best of our knowledge, this is the largest study to have evaluated TR late after left-sided valve surgery with a longterm echocardiographic follow-up.

\section{Clinical significance of late TR}

The development of late TR is a common and important manifestation after left-sided valve surgery and is known to be linked to exercise intolerance and a poor prognosis. ${ }^{6,15,16}$ In particular, moderate and severe TR are closely coupled with a poor prognosis, even in the absence of the left ventricular dysfunction or pulmonary hypertension. ${ }^{15}$ The reported incidences of TR late after left-sided valve surgery vary from $16 \%$ to $67 \%$, according to study population, the definition of significant TR, and follow-up duration. ${ }^{5,10-12}$ Tricuspid regurgitation incidence was found to be $16 \%$ by Matsuyama et $\mathrm{al}^{11}$, but the follow-up duration was relatively short (mean 8.2 years). In contrast, Porter et $\mathrm{al}^{5}$ reported a much higher incidence of $67 \%$ after a mean follow-up of ca 11 years, but the study population was relatively small. In the present study, the incidence of late TR development was found to be ca $27 \%$ with a mean follow-up of 11.6 years, and late TR was found to be closely associated with poor event-free survival. In our opinion, a follow-up duration of $>10$ years is required to detect patients who develop clinically significant TR late after left-sided valve surgery and to elucidate its prognostic implications. This is supported by the results shown in Figure 2, where the discrepancy in event-free cumulative survival rates was progressively widened 10 years after left-sided valve surgery.

\section{Mechanism of late TR}

Although the underlying mechanism of late TR after valve surgery is substantially unknown, it is generally considered "functional" in nature, because the tricuspid valve is morphologically normal in most patients. However, in contrast to the wealth of information available regarding functional mitral regurgitation, little is available on the mechanism of functional TR. In a previous study, we demonstrated that right ventricular geometry and tricuspid annular dilation are closely associated with functional TR development and severity. ${ }^{10,17,18}$ This issue requires further investigation.

\section{Prevention of late TR development}

In view of the clinical significance of TR, the identification of predictors of late TR is clinically important. Previous studies have shown that the presence of preoperative significant TR, the presence of preoperative atrial fibrillation, an advanced age, or huge left atrium is an independent predictor of late TR development. ${ }^{5,11,12}$ In the present study, we only recruited patients without significant preoperative TR to emphasize the de novo development of significant TR and to remove potential by residual TR after left-sided valve surgery. As a result, it was found that only 1 clinical factor, the presence of preoperative atrial fibrillation, contributed independently to the occurrence of late TR by multivariate analysis.

As corrective surgery for late TR is associated with high operative mortality and morbidity, ${ }^{8,9,19}$ its prevention has clinically important implications. Currently, tricuspid annuloplasty is widely performed as a safe and effective 
surgical tool for reducing tricuspid annular dimension, but no generally accepted indications for tricuspid annuloplasty have been established. Although preoperative TR severity and tricuspid annular dimension usually serve as a guide as to whether tricuspid valve annuloplasty is indicated, ${ }^{20}$ tricuspid annuloplasty is adamantly unsuccessful in a substantial number of patients. ${ }^{21}$

Our findings as is do not provide mechanistic insights into the determinants of late TR. The association with atrial fibrillation may be due to the fact that atrial fibrillation reflects a more advanced underlying valve disease. However, based on the previous observations on the impact of chronic atrial fibrillation, ${ }^{22-24}$ it is likely that chronic atrial fibrillation induces atrial remodeling and tricuspid annular dilation which, in turn, develops late TR. Therefore, it is suggested that tricuspid annuloplasty should be considered even in patients without significant TR in the presence of preoperative atrial fibrillation. In addition, a combined maze operation on top of tricuspid annuloplasty may provide a solution to the problem. Previously, we demonstrated that the maze operation can effectively prevent TR development due to the restoration and maintenance of atrial mechanical activity. ${ }^{25}$ More importantly, careful monitoring for the development of atrial fibrillation and the timely surgical correction of underlying left-sided valve disease may help prevent significant TR development late after surgery and possibly improve long-term prognosis.

\section{Limitations}

Several limitations of the present study need to be acknowledged. First, this study is limited by its retrospective nature. However, the preoperative, operative, and postoperative findings were prospectively summarized in our database system, which was specifically designed and maintained throughout the study period. Second, TR grade was not quantified, but although quantification may have provided an objective means of evaluating TR, semi-quantitative TR evaluations were well validated with angiographic evaluation and clinical measures of regurgitant severity and thus are widely used in practice. ${ }^{13,14}$ Finally, because we excluded 40 patients who died within 5 years of surgery to focus on the late development of TR, the event-free survival rates of our study population were artificially elevated and do not represent the true postoperative event-free survival.

\section{Conclusions}

The de novo development of significant TR long after left-sided valve surgery is not uncommon, with an estimated incidence of $27 \%$, and is closely associated with a poor prognosis. The present study identified preoperative atrial fibrillation as the only independent predictor for the development of late TR. Therefore, tricuspid annuloplasty should be considered in the presence of atrial fibrillation, even in patients' mild TR at the time of surgical correction for left-sided valve disease.

\section{References}

1. Czer LS, Maurer G, Bolger A, et al. Tricuspid valve repair. Operative and follow-up evaluation by Doppler color flow mapping. J Thorac Cardiovasc Surg 1989;98:101-10.

2. Goldman ME, Guarino T, Fuster V, et al. The necessity for tricuspid valve repair can be determined intraoperatively by two-dimensional echocardiography. J Thorac Cardiovasc Surg 1987;94:542-50.

3. Hannoush H, Fawzy ME, Stefadouros M, et al. Regression of significant tricuspid regurgitation after mitral balloon valvotomy for severe mitral stenosis. Am Heart J 2004;148:865-70.

4. McGrath LB, Gonzalez-Lavin L, Bailey BM, et al. Tricuspid valve operations in 530 patients. Twenty-five-year assessment of early and late phase events. J Thorac Cardiovasc Surg 1990;99:124-33.

5. Porter A, Shapira $Y$, Wurzel $M$, et al. Tricuspid regurgitation late after mitral valve replacement: clinical and echocardiographic evaluation. J Heart Valve Dis 1999;8:57-62.

6. Groves PH, Lewis NP, Ikram S, et al. Reduced exercise capacity in patients with tricuspid regurgitation after successful mitral valve replacement for rheumatic mitral valve disease. $\mathrm{Br}$ Heart J $1991 ; 66: 295-301$.

7. Koelling TM, Aaronson KD, Cody RJ, et al. Prognostic significance of mitral regurgitation and tricuspid regurgitation in patients with left ventricular systolic dysfunction. Am Heart J 2002;144:524-9.

8. King RM, Schaff HV, Danielson GK, et al. Surgery for tricuspid regurgitation late after mitral valve replacement. Circulation 1984;70:1193-1197.

9. Staab ME, Nishimura RA, Dearani JA. Isolated tricuspid valve surgery for severe tricuspid regurgitation following prior left heart valve surgery: analysis of outcome in 34 patients. J Heart Valve Dis 1999;8:567-74.

10. Groves PH, Ikram S, Ingold U, et al. Tricuspid regurgitation following mitral valve replacement: an echocardiographic study. J Heart Valve Dis 1993;2:273-8.

11. Matsuyama K, Matsumoto M, Sugita T, et al. Predictors of residual tricuspid regurgitation after mitral valve surgery. Ann Thorac Surg 2003;75:1826-8.

12. Izumi C, Iga K, Konishi T. Progression of isolated tricuspid regurgitation late after mitral valve surgery for rheumatic mitral valve disease. J Heart Valve Dis 2002;1 1:353-6.

13. Zoghbi WA, Enriquez-Sarano M, Foster E, et al. Recommendations for evaluation of the severity of native valvular regurgitation with twodimensional and Doppler echocardiography. J Am Soc Echocardiogr 2003;16:777-802.

14. Singh JP, Evans JC, Levy D, et al. Mitral, tricuspid, and aortic regurgitation (The Framingham heart study). Am J Cardiol 1999;83:897-902.

15. Groves PH, Hall RJ. Tricuspid regurgitation following mitral valve surgery. J Heart Valve Dis 1992;1:80-6.

16. Nath J, Foster E, Heidenreich PA. Impact of tricuspid regurgitation on long-term survival. J Am Coll Cardiol 2004;43:405-9.

17. Kim HK, Kim YJ, Park JS, et al. Determinants of the severity of functional regurgitation. Am J Cardiol 2006;98:236-42.

18. Fukuda $S$, Gillinov $A M$, Song JM, et al. Echocardiographic insights into atrial and ventricular mechanisms of functional tricuspid regurgitation. Am Heart J 2006;152:1208-14.

19. Kwon DA, Park JS, Chang HJ, et al. Prediction of outcome in patients undergoing surgery for severe tricuspid regurgitation following mitral valve surgery and role of tricuspid annular systolic velocity. Am J Cardiol 2006;98:659-61. 
20. Colombo T, Russo C, Ciliberto GR, et al. Tricuspid regurgitation secondary to mitral valve disease: tricuspid annulus function as guide to tricuspid valve repair. Cardiovasc Surg 2001;9:369-77.

21. Fukuda S, Song JM, Gillinov AM, et al. Tricuspid valve tethering predicts residual tricuspid regurgitation after tricuspid annuloplasty. Circulation 2005; 111:975-9.

22. Vaturi M, Sagie A, Shapira $Y$, et al. Impact of atrial fibrillation on clinical status, atrial size and hemodynamics in patients after mitral valve replacement. J Heart Valve Dis 2001;10:763-6.
23. Obadia JF, el Farra M, Bastien $\mathrm{OH}$, et al. Outcome of atrial fibrillation after mitral valve repair. J Thorac Cardiovasc Surg 1997; 114:179-85.

24. Flugelman MY, Hasin $Y$, Katznelson $N$, et al. Restoration and maintenance of sinus rhythm after mitral valve surgery for mitral stenosis. Am J Cardiol 1984;54:617-9.

25. Kim HK, Kim YJ, Kim KI, et al. Impact of the maze operation combined with left-sided valve surgery on the change in tricuspid regurgitation over time. Circulation 2005;112:114-9. 